\title{
Time-dependent convection at high latitudes
}

\author{
D. W. Idenden ${ }^{1}$, R. J. Moffett ${ }^{1}$, S. Quegan ${ }^{1}$, T. J. Fuller-Rowell ${ }^{2}$ \\ ${ }^{1}$ School of Mathematics and Statistics, The Hicks Building, University of Sheffield, Sheffield, S3 7RH, UK \\ 2 CIRES, University of Colorado/NOAA Space Environment Laboratory, 325 Broadway, Boulder, CO 80303, USA
}

Received: 12 January 1996/Revised: 20 May 1996/Accepted: 27 May 1996

\begin{abstract}
A fully time-dependent ionospheric convection model, in which electric potentials are derived by an analytic solution of Laplace's equation, is described. This model has been developed to replace the empirically derived average convection patterns currently used routinely in the Sheffield/SEL/UCL coupled thermosphere/ionosphere/plasmasphere model (CTIP) for modelling disturbed periods. Illustrative studies of such periods indicate that, for the electric field pulsation periods imposed, longterm averages of parameters such as Joule heating and plasma density have significantly different values in a time-dependent model compared to those derived under the same mean conditions in a steady-state model. These differences are indicative of the highly non-linear nature of the processes involved.
\end{abstract}

\section{Introduction}

The processes of magnetic field connection and reconnection in the magnetosphere, first suggested by Dungey (1961), are now well established as the primary driving mechanisms for the two-cell convection pattern observed during periods of strong southward interplanetary magnetic field (IMF). When the high velocity solar wind consisting of a hot magnetised plasma interacts with the magnetosphere, the whole system can be viewed as an enormous magnetohydrodynamic generator. A proportion of the large voltage (hundreds of $\mathrm{kV}$ ) applied in the dawn-dusk direction across the magnetopause is mapped down into the ionosphere along highly conducting geomagnetic field lines when the latter become connected to the IMF, usually close to the subsolar point on the magnetopause. These connected, 'open' field lines have only one foot on the Earth and are swept into the tail of the magnetosphere by the solar wind. The electric field in

Correspondence to: D. W. Idenden the ionosphere gives rise to an anti-sunward $\mathbf{E} \times \mathbf{B}$ drift across the polar cap which may be defined as the area enclosing these open field lines. Open field lines swept and stretched into the tail lobes will, at some stage, reconnect with their partners in the opposite hemisphere and return sunward under magnetic tension. These closed field lines map a dawn-dusk field into the ionosphere where the sunward convection is mirrored and a two-cell convection pattern arises.

Under steady-state conditions, snapshots of ionospheric convection taken at different instants in time will be identical. Thus, the polar cap boundary separating anti-sunward convecting plasma on open field lines from sunward convecting plasma on closed field lines will remain fixed. This condition implies that, since the magnetic flux is incompressible, plasma flowing into the polar cap through the dayside throat must be exactly balanced by the flow of plasma through the nightside exit region.

Most empirical models developed to describe ionospheric convection are, by the nature of the method of their development, inherently steady-state. Such models make extensive use of satellite data (Heppner, 1977; Heppner and Maynard, 1987), radar data (Foster et al., 1986; Holt et al., 1987) or a combination of both (Heelis et al., 1983). In addition, Friis Christensen et al. (1985) describe how a global map of ionospheric convection is inferred from Greenland magnetometer data. Generally data is binned according to concurrent IMF conditions. Foster et al. (1986), however, bin their data according to the total amount of energy in precipitating particles inferred from TIROS satellite measurements. Convection patterns are obtained for each dataset by a superposition and statistical averaging of many measurements spanning many hours, days or even months of observations. These models, then, should not be treated as snapshots of convection. The assumption that, at any given state of the solar wind field, a corresponding steady-state convection pattern prevails is most certainly flawed. In the event that the dayside and nightside merging rates do indeed depend only on the IMF conditions, there would nevertheless be an inevitable delay between the occurrence of a change in 
the solar wind field at the dayside magnetopause and the transfer of such information many Earth radii into the tail of the magnetosphere where reconnection occurs. Lockwood (1991) suggests that it may take in the region of $2 \mathrm{~h}$ of more or less constant IMF conditions to prevail before steady-state convection is achieved. Such events are rare, occurring only $15 \%$ of the time (Rostoker et al., 1988). Additional complications arise in the form of substorms whereby solar wind energy is stored in the magnetotail and released explosively.

Thus, in general, empirical models and their analytical fits (for example Hairston and Heelis, 1990) must be regarded as average convection patterns. A snapshot of the instantaneous pattern may look very different from these empirical models and even gross mean features may be lost by the smearing effect of the statistical averaging process. Holt et al. (1987) report the loss of such large features as the Harang discontinuity which is clearly seen on individual days but disappears in averages over large data sets. The recent analysis procedure, AMIE (Richmond, 1992) has gone some way to solving this problem. The technique involves performing a least squares fit of an analytic electric potential distribution to data obtained simultaneously by many different instruments (i.e. satellite, magnetometer and radar), though some assumptions regarding ionospheric conductivity need to be made in order to obtain electric fields from the derived current system.

The model described in this study is based on an analytic solution of Laplace's equation on a plane surface which was originally solved for non-steady-state reconnection by Siscoe and Huang (1985) who imposed boundary conditions by specifying the potential around the polar cap boundary only. Flux is considered to enter and leave the polar cap through only the throat and night exit regions respectively. The throat maps to the merging line at the dayside magnetopause where connection to the IMF occurs, while the night exit maps to the region where field lines close in the neutral sheet. The remainder of the polar cap boundary is considered adiaroic, i.e., no plasma may flow across it.

Siscoe and Huang's solution was restricted to dayside merging only. Thus, the polar cap expanded continuously as plasma flowed through the throat. Our model represents an improvement over the Siscoe and Huang formulation in a number of respects: (1) both dayside and nightside merging may be simulated; (2) the latitudinal extent of convection may be restricted by imposing a second boundary beyond which the electric potential falls to zero. Such a restriction enables better fits to be obtained to empirical models such as those of Heppner and Maynard (1987) and Foster et al. (1986) and takes better account of theoretical arguments which invoke shielding by the ring current to prevent penetration of the electric field to lower latitudes (for example Wolf, 1975); (3) dawndusk asymmetries may be produced by (a) placing throat and exit regions asymmetrically and (b) adding a monopole term (which has only a radial electric field component and thus adds a vortex component to the flow lines) to the solution. These refinements allow for the possibility of extending the model to allow for flows observed when the $B_{y}$ component of the IMF is non-zero.
In using a solution of Laplace's equation with the type of boundary conditions we have described, we have essentially made two implicit assumptions; (1) that the conductivities are uniform within the area of interest and (2) that field aligned currents are restricted to the polar cap and zero potential boundaries only. While the first assumption is evidently not true, Moses et al. (1994) found that plasma flows during a geomagnetic storm could be modelled more accurately by assuming uniform conductivity than by imposing a highly conducting auroral oval or day/ night conductivity gradient. The effect of the former would only be significant near to the throat and night exit regions (the only regions where there is communication between polar cap and auroral plasma). In these areas flow lines would be kinked while gradients across the terminator tend to concentrate flow lines in the dawn sector. Nevertheless the two cell convection pattern persists for strongly southward IMF. Iijima and Potemra (1978) showed that large-scale region 1 and region 2 current systems were restricted to the poleward and equatorward edge of the field aligned current region in the auroral oval at all magnetic local time sectors apart from a few hours pre-midnight where current regions overlap in a more complex fashion. Thus, although indeed the majority of current flows along the surface of two concentric cylinders, there are significant field aligned currents across much of the auroral ionosphere. The justification for our approach here is that the flow patterns we obtain under steady-state conditions agree well with average empirical models.

Numerical solutions to Laplace's equation have also been developed by Moses et al. (1987, 1989). Boundary conditions are set along a spiral polar cap boundary which allows modelling of large zonal flows near the merging gaps of the types observed during periods when the magnitude of the IMF $B_{y}$ component is large. While no direct observational evidence would suggest a spiral shape for the polar cap boundary, good fits to many single pass satellite measurements during a geomagnetic storm have been obtained with this model (Moses et al., 1994).

\section{Model description}

Assuming that ionospheric currents perturb the Earth's magnetic field only slightly, we can take $\nabla \times \mathbf{E}=0$ and hence define the electric field $\mathbf{E}$ by a scalar potential $\Phi$ where, by convention, $\mathbf{E}=-\nabla \Phi$. For the purpose of describing the analytic electric field model, we need first to define a coordinate system whose origin is at the centre of the polar cap (Fig. 1). The model electric potentials are those that will give the desired $\mathbf{E} \times \mathbf{B}$ velocities when measured in the rest frame of this system. Azimuth is measured anti-clockwise from the meridian that passes through the geomagnetic pole while $r$ is measured from the coordinate origin. Following Siscoe and Huang (1985), the potential must first be specified around the polar cap boundary. In the steady-state case, the adiaroic segments of the boundary are equipotentials while the potential varies linearly along them when merging is unbalanced. Dayside and nightside merging rates are controlled by 


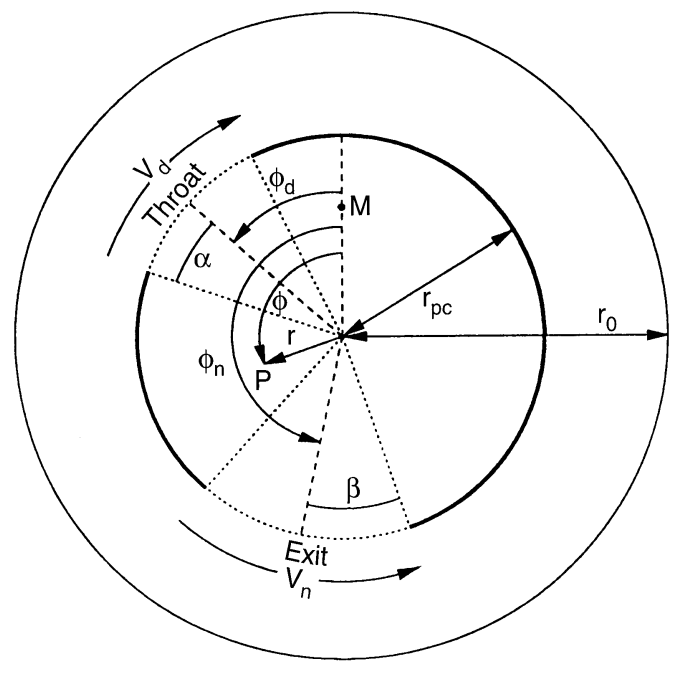

Fig. 1. The geometry of the polar cap. The geopotential coordinates $(\phi, r)$ are shown for the point $P$. The geopotential pole is at the centre of the polar cap, and the geomagnetic pole is marked $M$. The bold lines mark the adiaroic sections of the polar cap boundary. The outer boundary shows the extent of the high-latitude convection pattern. $\phi_{d}$ and $\phi_{n}$ are the azimuths of the centre of the throat and night exit regions respectively which have half-widths of $\alpha$ and $\beta$ respectively. $V_{d}$ and $V_{n}$ are the voltages measured across the two gaps

specifying the potential differences across the throat and night exit regions, $V_{d}$ and $V_{n}$, respectively. Any difference between $V_{d}$ and $V_{n}$ is distributed uniformly around the adiaroic boundary segments. The potential in the flux gaps takes the form:

$\Phi\left(r=r_{p c}, \phi\right)=a\left(\frac{\phi-\phi_{0}}{\alpha}\right)+b\left(\frac{\phi-\phi_{0}}{\alpha}\right)^{3}+c$,

where $\alpha$ is the flux gap half-width, $r_{p c}$ is the polar cap radius, $\phi_{0}$ is the azimuth of the centre of the flux gap (equal to $\phi_{d}$ and $\phi_{n}$ for the dayside and nightside gaps respectively), and $a, b$ and $c$ are calculated to obtain the required potential drop across the gap and uniformity in $\partial \Phi / \partial \phi$ around the boundary. The coefficient $c$ is required for flux gaps positioned asymmetrically on the boundary, though it may arbitrarily be set to zero for either one of the gaps. The general solution of Laplace's equation may be written:

$$
\begin{aligned}
\Phi(r, \phi)= & \sum_{n=1}^{\infty}\left(\frac{r}{a}\right)^{n}\left(A_{n} \cos (n \phi)+B_{n} \sin (n \phi)\right) \\
& +\sum_{n=1}^{\infty}\left(\frac{r}{a}\right)^{-n}\left(C_{n} \cos (n \phi)+D_{n} \sin (n \phi)\right) \\
& +E \ln (r)+F .
\end{aligned}
$$

The coefficients, $A_{n}, B_{n}, C_{n}$, and $D_{n}$, may be obtained by imposing the conditions at the polar cap boundary and at the zero potential boundary. $E \ln (r)$ is the term referred to previously as the monopole term. The Fourier coefficients for the potential along the polar cap boundary are given by:

$\alpha_{n}=\frac{1}{\pi} \int_{0}^{2 \pi} \sin (n \phi) \Phi\left(r=r_{p c}, \phi\right) d \phi$

and

$\beta_{n}=\frac{1}{\pi} \int_{0}^{2 \pi} \cos (n \phi) \Phi\left(r=r_{p c}, \phi\right) d \phi$.

The solution now takes different forms in the three regions $r<r_{p c}, r_{p c}<r<r_{0}$ and $r>r_{0}$ where $r_{0}$ is the radius at which the potential falls to zero.

\subsection{In the auroral oval, $r_{p c}<r<r_{0}$}

Continuity in the potential at the polar cap implies the conditions

$A_{n}+C_{n}=\alpha_{n}$

and

$B_{n}+D_{n}=\beta_{n}$.

Additionally, the boundary condition $\Phi\left(r_{0}\right)=0$ requires that

$\left(\frac{r_{0}}{r_{p c}}\right)^{n} A_{n}+\left(\frac{r_{0}}{r_{p c}}\right)^{-n} C_{n}=0$

and

$\left(\frac{r_{0}}{r_{p c}}\right)^{n} B_{n}+\left(\frac{r_{0}}{r_{p c}}\right)^{-n} D_{n}=0$

are satisfied for all $n$. Thus, solving for the unknowns $A_{n}$, $B_{n}, C_{n}$ and $D_{n}$ in Eqs. (5-8) gives

$$
\begin{aligned}
& A_{n}=\frac{\alpha_{n}}{1-\left(\frac{r_{0}}{r_{p c}}\right)^{2 n}}, \\
& B_{n}=\frac{\beta_{n}}{1-\left(\frac{r_{0}}{r_{p c}}\right)^{2 n}}, \\
& C_{n}=\frac{\alpha_{n}}{1-\left(\frac{r_{p c}}{r_{0}}\right)^{2 n}}, \\
& D_{n}=\frac{\beta_{n}}{1-\left(\frac{r_{p c}}{r_{0}}\right)^{2 n}} .
\end{aligned}
$$

$E$ may be chosen to fit data or empirical models and $F$ can be adjusted to ensure that the condition $\Phi\left(r_{0}\right)=0$ is satisfied:

$F=-E \ln \left(\frac{r_{0}}{r_{p c}}\right)$. 


\subsection{Inside the polar cap, $r<r_{p c}$}

The requirement that the electric potential be finite within the polar cap, and in particular at the origin, allows us to drop many of the terms in Eq. (2):

$C_{n}^{\prime}=D_{n}^{\prime}=E_{n}^{\prime}=0$,

where' denotes the area inside the polar cap boundary. Thus, within the polar cap, the potential is given by:

$\Phi(r, \phi)=\sum_{n=1}^{\infty}\left(\frac{r}{a}\right)^{n}\left(A_{n}^{\prime} \cos (n \phi)+B_{n}^{\prime} \sin (n \phi)\right)+F^{\prime}$.

Continuity in $\Phi$ at the boundary enables us to determine the remaining coefficients:

$A_{n}^{\prime}=\alpha_{n}$,

$B_{n}^{\prime}=\beta_{n}$,

$F^{\prime}=F$.

\subsection{Outside the high-latitude region, $r>r_{0}$}

The magnetospheric potential is set to zero at all points. The dynamo effect generates electric fields at lower latitudes. These are not considered here.

\section{Correction for corotation}

The electric field model described so far enables a two-cell convection pattern to be generated in the corotating frame as measured by an Earth-bound observer or after transformation of satellite measurements by removing the corotation velocity. The underlying assumption in defining the potential along the polar cap boundary, essentially the boundary conditions used to solve Laplace's equation, is that the polar cap boundary is stationary in the corotating frame (and thus an equipotential under steady-state conditions). However, results obtained by fitting circular convection reversal boundaries to potential extrema from satellite observations (Meng et al., 1977; Hairston and Heelis, 1990) would indicate that the centre of the polar cap is displaced a few degrees to the nightside of the geomagnetic pole. These observations are also confirmed by fitting convection reversal boundaries to the empirical models of Foster et al. (1986) which are described later in this study. Thus, the polar cap boundary is stationary in the sun-fixed geomagnetic frame (i.e. that defined by MLT and invariant latitude). The boundary conditions we have described i.e. an equipotential under steady-state conditions or linearly varying for an expanding/contracting polar cap are relevant to this frame. To obtain the potential function along the boundary in the corotating frame, we must make a correction by subtracting the 'corotation potential function', $\Phi_{M C}$, which describes the motion of corotating plasma in the Sun-fixed geomagnetic frame. Quegan et al. (1986) showed $\Phi_{m c}$ to depend on invariant latitude and UT only, the UT dependence arising from the offset of the geomagnetic and geographic poles. The corotation potential measured around the boundary is a cosine
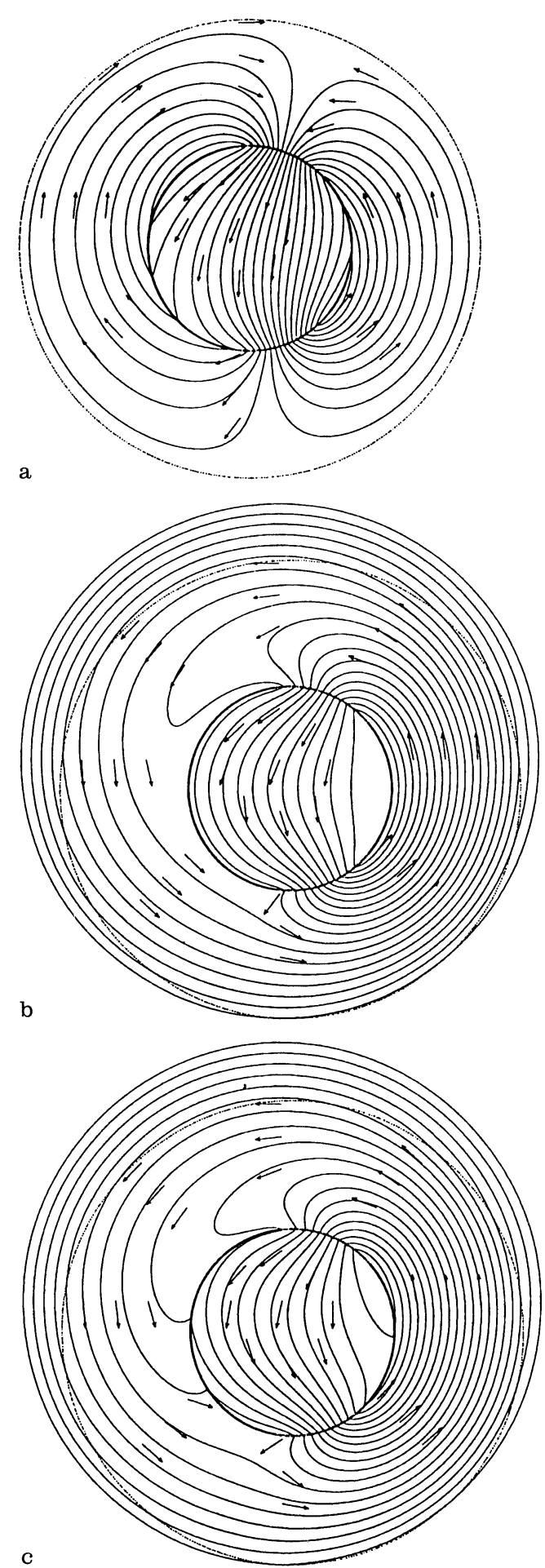

Fig. 2a-c. Plots of equipotentials (plasma flow lines). Arrows indicate flow direction. a Steady-state convection in the corotating frame; b steady-state convection in the Sun-fixed (non-corotating) geomagnetic frame; $\mathbf{c}$ as $\mathbf{b}$, but omitting correction to boundary potential to force the boundary to be stationary in this frame. Flow is seen across adiaroic boundary segments (bold lines). The dotdashed line marks the extent of the high-latitude convection pattern. Noon MLT is at the top of these plots 
function of azimuth measured in the geopotential coordinate system plus a constant offset potential. The offset may be ignored since it is only the potential gradient which is of interest for plasma dynamics. Thus,

$\Phi_{m c}\left(\phi, r=r_{p c}, \mathrm{UT}\right)=A_{c}\left(r_{p c}, \mathrm{UT}\right) \cos \phi$.

The amplitude of the corotation potential around the boundary is given by:

$$
\begin{aligned}
A_{c}\left(r_{p c}, \mathrm{UT}\right)= & k \frac{\cos \left(\theta_{m}\right)-\sin (\delta) \sin \left(\delta^{\prime}\right)}{1-\sin ^{2}(\delta)} \\
& \times\left[\frac{\sin ^{2}\left(r_{p c}-\theta_{p}\right)-\sin ^{2}\left(r_{p c}+\theta_{p}\right)}{2}\right],
\end{aligned}
$$

where $\theta_{m}$ is the separation of the geomagnetic and geographic poles, $\theta_{p}$ is the separation of the geomagnetic pole and the polar cap centre, $\delta$ and $\delta^{\prime}$ are the Sun's declinations in the geographic and Sun-fixed geomagnetic frames respectively (the latter being implicitly UT dependent), and $k \approx-84543 \mathrm{~V}$. Now it is a straightforward matter to subtract the corotation potential from the boundary by adjusting the first cosine Fourier coefficient, $\alpha_{1}$ :

$\alpha_{1}=\alpha_{1}^{\prime}-A_{c}\left(r_{p c}, \mathrm{UT}\right)$,

where $\alpha_{1}^{\prime}$ is the coefficient obtained in the geopotential rest frame.

Figure 2a shows an example of the resulting steadystate convection pattern in the corotating frame. The adiaroic boundary is no longer an equipotential since the boundary itself is moving in this frame. Figure $2 b$ shows the same pattern in the Sun-fixed geomagnetic frame in which the boundary is stationary and thus an equipotential. This may be contrasted with Fig. 2c in which no correction term has been included in the electric field and consequently flow lines can be seen passing through the stationary 'adiaroic' boundary segments. Figure 3a, b shows typical non-steady-state convection patterns with the inclusion of the correction term for expanding and contracting polar caps respectively.

\section{Non-zero $B_{y}$ fields - a possible extension to the model}

The model described so far is considered to present a significant improvement over the analytic model described by Siscoe and Huang (1985) and thus, an appropriate starting place in time dependent modelling of the highlatitude ionosphere/thermosphere system in a self-consistent regime. Nevertheless, flows resulting from a non-zero $B_{y}$ component of the IMF have not been included in the model, though these are considered briefly below where we suggest ways in which they might be incorporated into the analytic field.

Non-zero $B_{y}$ fields are characterised by a number of features in the ionospheric flow paths which arise from field line tension and may be summarised for the Northern Hemisphere as follows:

a. The dayside cusp is displaced toward dusk (dawn) for positive (negative) By (Lockwood et al., 1990; Reiff and Burch, 1985). b. The convection cells become asymmetric. For positive (negative) $B_{y}$, the dawn (dusk) cell is kidney-shaped and partially surrounds the larger dusk (dawn) cell. The electric field and hence convection is enhanced on the dawn (dusk) side.

c. The geometry described results in strong zonal flows within the polar cap which are westward (eastward) for
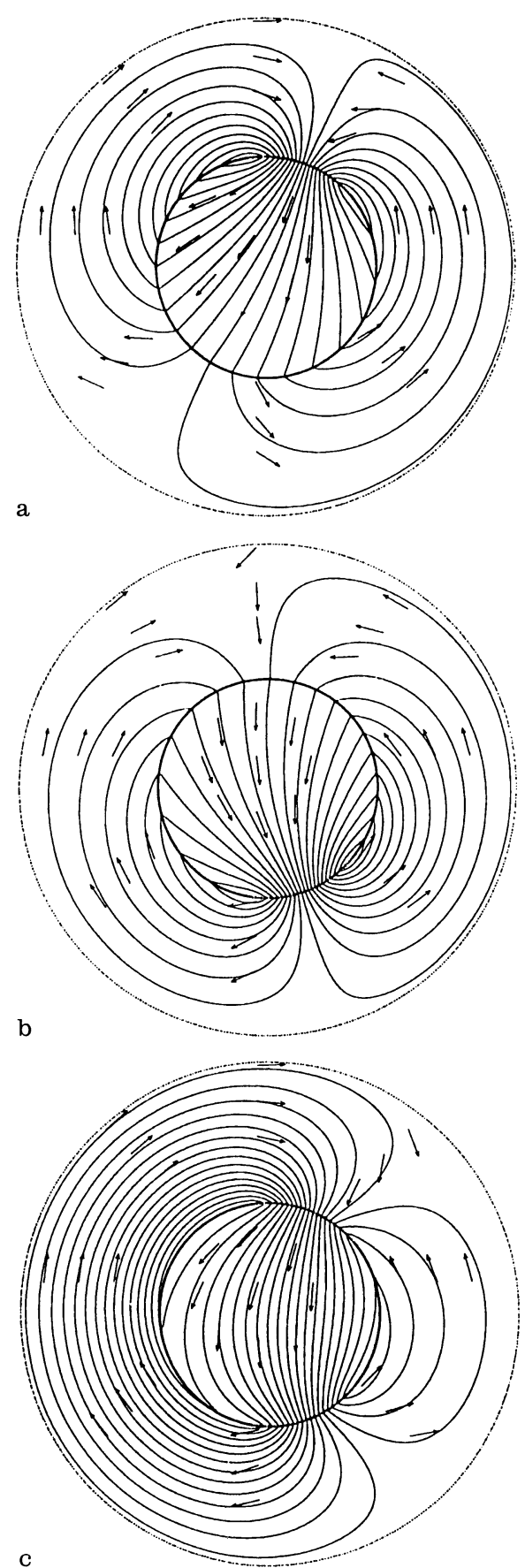

Fig. 3a-c. Snapshots of equipotential plasma flow lines in the Sunfixed geomagnetic frame. a Expanding polar cap convection pattern; b contracting polar cap; c steady-state convection pattern with $B_{y}$-type flows: circulation within the polar cap and asymmetric dawn/dusk cells. Noon MLT is at the top of these plots 
positive (negative) $B_{y}$. Moses et al. (1987, 1989) model $B_{y}$ flows using a numerical scheme for solving Laplace's equation with a spiral polar cap boundary. In the analytic model described here, a number of refinements can be added to simulate such flows: (a) the position of the dayside throat can be moved duskward/dawnward depending on the required value of $B_{y}$; (b) the monopole term affecting flows outside the boundary may be adjusted to produce kidney shaped convection cells; (c) within the polar cap, a circulation term may be added to produce zonal flows as suggested by Siscoe and Huang (1985). A circulation term which by itself would produce flow of constant angular velocity around the polar cap (similar to the way in which the corotation potential produces flow around the geographic poles in the Sun-fixed frame) is not actually a solution to Laplace's equation and would therefore require a continuous distribution of field-aligned Birkeland currents to support it.

The effect of these refinements is shown in Fig. 3c. When the circulation term is of similar magnitude to the cross polar cap potential drop, a lobe cell is formed within the polar cap which has been observed during weak southward $B_{z}$ and strong $B_{y}$ (Reiff and Burch, 1985). Such flows are attributed to lobe stirring due to reconnection of already open field lines with the interplanetary magnetic field (Crooker, 1979). During periods of northward IMF when dayside merging disappears altogether, flows will be dominated by this lobe stirring, nightside reconnection and viscous flow.

\section{Model summary}

The model described uses an analytic solution of Laplace's equation on a two-dimensional plane and is a good approximation to a solution on a spherical surface for latitudes close to the poles. The model requires as inputs: the initial polar cap radius; the voltages across the dayside and nightside merging gaps, each as functions of time; the throat and exit widths and positions on the polar cap boundary (also functions of time); and the magnitude of the monopole term. While some dawn-dusk asymmetry is permitted in the flow by moving the positions of the merging gaps, and including a monopole term outside the polar cap, these adjustments are at present used only to obtain better fits to empirical models. We suggest means by which non-zero $B_{y}$ flows may be modelled in the analytic framework.

\section{Effect on the ionosphere of time-dependent convection}

To test the effects of time varying electric fields on the high-latitude ionosphere, the time-dependent convection model has been incorporated into the Sheffield/SEL/UCL coupled thermosphere/ionosphere/plasmasphere model (CTIP).

\subsection{Incorporating time-dependent convection into CTIP}

The time-dependent convection model has replaced the empirical model of Foster et al. (1986) in a development version of the CTIP (Millward et al., 1995) with the intention of: (1) comparing average results obtained using time varying electric fields with those from a steady-state field to determine the shortcomings of steady-state models in predicting mean values of ionospheric and thermospheric parameters; (2) looking for transient effects produced by pulsed electric fields.

The empirical models of Foster are matched to maps of precipitation determined by the TIROS-NOAA satellites (Fuller-Rowell and Evans, 1987), each parametrised by an index denoting the mean power of precipitation. Appropriate steady-state conditions for the present model were determined by fits to the electric fields obtained from the Foster empirical model at each precipitation level. Fits were obtained by a least squares method in which the position of the polar cap centre, polar cap radius, zero potential radius $\left(r_{0}\right)$, position of merging gaps, monopole amplitude and cross-polar-cap voltage were optimised. Constraints on fits were that the dayside and nightside potential differences remained equal (the condition of steady-state) and the widths of the merging gaps remained at $90^{\circ}$. Relaxation of this latter condition results in the gaps growing much larger. This is due to the rotational shears apparent around much of the polar cap boundary in the empirical models. Such flows may, in part, arise from viscous flows which would produce rotational shears around the whole polar cap boundary but are not considered by our model. Apparent rotational shears may also be generated in empirical models derived by averaging over periods when the polar cap is expanding and contracting over its mean location (Lockwood, 1991). Additionally, radars employing beam swinging techniques may measure rotational reversals where shear reversals actually exist due to assumptions of spatial uniformity of flow employed to make such measurements (Lockwood et al., 1988). Single-pass satellite measurements do, however, tend to show shear reversal on large sections of the polar cap boundary, implying the presence of adiaroic segments and confined throat and night exit regions (Heelis et al., 1976, 1982, Heelis and Hanson, 1980). Plots of equipotentials for the empirical models are shown in Fig. 4 along with the fits for the present model for precipitation levels 5 and 9 .

\subsection{Dealing with precipitation in time-varying convection}

Precipitation during time-varying conditions has been calculated using the TIROS-NOAA precipitation patterns corresponding to the mean conditions during the period. The pattern as a whole is simply made to expand or contract in proportion to the radius of the polar cap. Such a time-varying precipitation model is used as a first attempt in the absence of appropriate empirical models. 

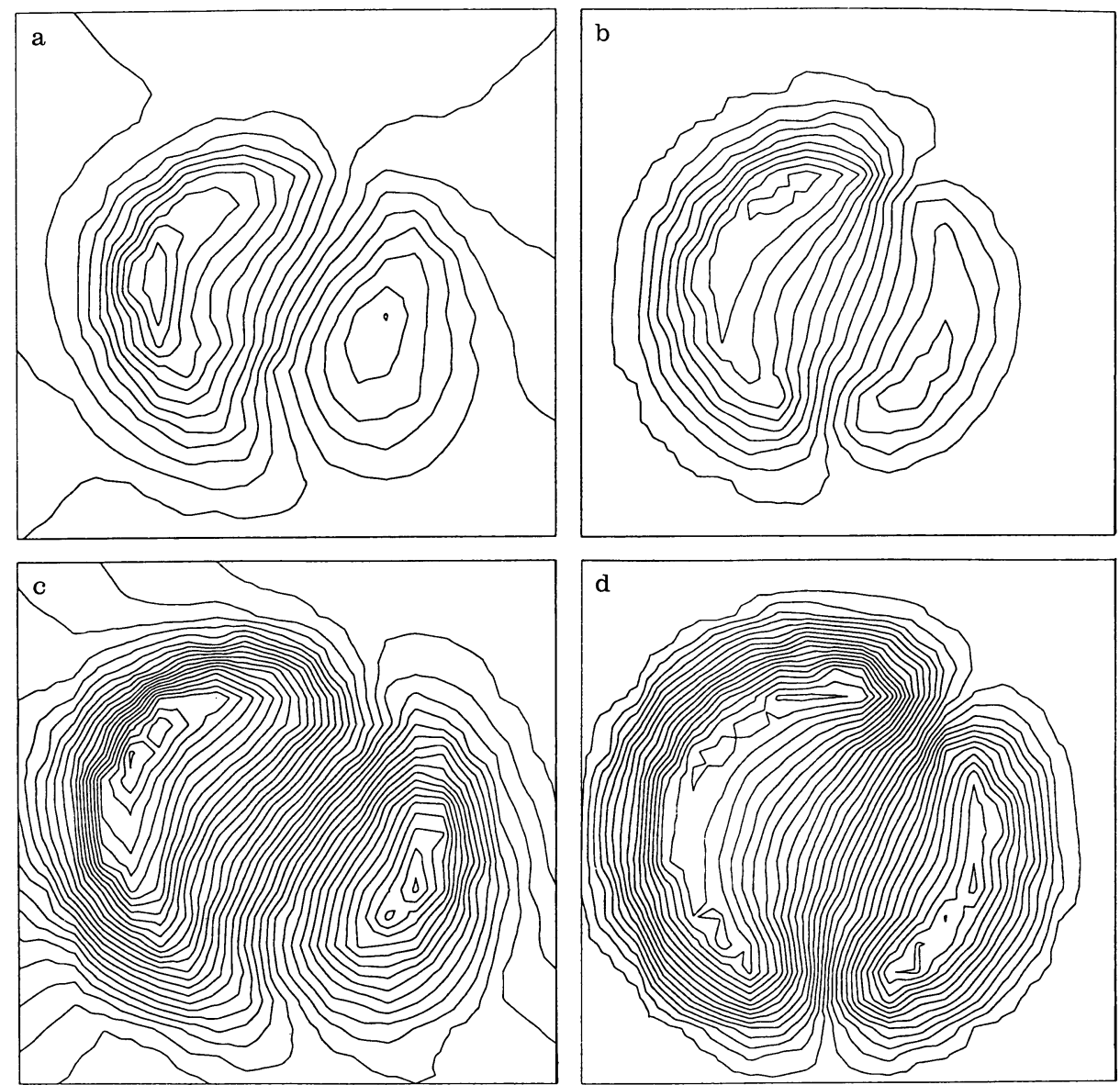

Fig. 4a-d. Plasma flow line equipotentials in the Sun-fixed geomagnetic frame for the Foster et al. (1986) model, a and c and the time- dependent model discussed in this work fitted to the Foster et al. (1986) model, $\mathbf{b}$ and $\mathbf{d}$. $\mathbf{a}$ and $\mathbf{b}$ are for activity level 5 , and $\mathbf{c}$ and $\mathbf{d}$ are for activity level 9. Noon MLT is at the top of these plots, and contours are at intervals of $2 \mathrm{kV}$

\section{Time-dependent and steady-state computations}

A comparison of results obtained using steady-state and time-dependent models with the same mean conditions (cross-polar-cap voltage, $V_{p c}$, and polar cap area) has been undertaken to determine the effect of non-steady convection on average values of thermospheric and ionospheric parameters and thus the limitations of steady-state models in determining accurate values of these parameters. In this context, $V_{p c}$ is defined as the dawn-dusk potential drop across the polar cap measured along any line which passes through the centre of the polar cap and intersects the adiaroic boundary in both the morning and afternoon sectors.

Both versions of CTIP were run for 24-h simulations to reach quasi-steady states before average values were obtained for parameters over a 1-h period for the area close to the northern polar cap. Three days contrasting greatly in plasma density distribution were chosen for study: winter solstice (day 355), summer solstice (172) and northern autumn equinox (263). Comparisons were made for both quiet-time mean conditions corresponding to activity level $5\left(K_{P}=2+, V_{p c}=29 \mathrm{kV}\right)$ and more disturbed conditions, activity level $9\left(K_{P}=5-, V_{p c}=66 \mathrm{kV}\right)$.

Time dependent electric fields are modelled by a polar cap expansion phase followed by a contraction about the desired mean polar cap area with a 1 -h period. During expansion, the nightside flux gap width and voltage are both set to zero, and the dayside voltage set to obtain the same polar cap voltage, $V_{p c}$, (as defined already) as in the steady state case $\left(V_{S S}\right)$. Since the potential varies linearly along the adiaroic boundary segment, which in this instance is $270^{\circ}$ in azimuthal extent, this latter requirement is satisfied by setting $V_{d}=\frac{3}{2} V_{S S}$. Similarly during contraction the dayside voltage and flux gap widths are set to zero, and $V_{n}=\frac{3}{2} V_{S S}$. Thus, dayside and nightside voltages have square wave forms and are $180^{\circ}$ out of phase with each other. Although somewhat arbitrary, these conditions do ensure that the plasma velocity through the exit region is never less than the boundary velocity. If this were not the case, previously closed flux tubes on the nightside could effectively become open, which is un-physical. The 1-h period is chosen as roughly the time scale for substorm activity. Though results obtained using other periods will not be presented here, preliminary examination of parameters obtained using a 0.5 -h period did not reveal any significant (i.e greater than a few percent) differences to those obtained with the 1 -h period. The polar cap radius is calculated self-consistently at each time step according to the rate of expansion/contraction due to the electric field along the adiaroic boundary segment.

Particular emphasis was placed on the study of Joule heating which is the primary mechanism by which energy is dissipated in the neutral atmosphere by convection. 
Complementary to this was the study of plasma densities which both influence Joule heating (since this depends directly on ionospheric conductivity), and are influenced by frictional heating of the ionosphere (equivalent to Joule heating) which can cause plasma depletion via temperature dependent loss reactions. Plasma convection also couples to the thermosphere via electrodynamic (Lorentz) forcing which results in momentum transfer to the neutral atmosphere giving rise to large-scale neutral winds. Neutral winds are also generated by thermal expansion of the thermosphere produced by Joule heating. These winds may have a component perpendicular to the Lorentz force which is predominantly in the direction of ion flow in the $F$ region. We report here a sample of our results for the Northern Hemisphere as illustrations of the effects of time dependent convection.

\subsection{Results}

Values for the time- and area-averaged rates of energy input by Joule heating into the thermosphere within the boundary defining the latitudinal extent of the high-latitude magnetospheric convection at F-region heights (pressure level 12, about $280 \mathrm{~km}$ ) have been calculated for steady-state and time-dependent models. These values are shown in Table 1. The largest difference is seen for the lower activity level at winter solstice when the time varying field produces nearly $120 \%$ more heating within the polar cap than the steady-state field. Joule heating enhancements of at least $80 \%$ within the polar cap are seen in all other datasets apart from during the lower activity level at summer solstice when this value is only $50 \%$. Enhancements in the annulus outside the polar cap are significantly less than inside the polar cap. At the lower

Table 1. Comparison of steady-state (SS) and time-dependent (TD) mean Joule heating rates for the high-latitude Northern Hemisphere region, pressure level 12 (approx. $280 \mathrm{~km}$ ), $\mathrm{W} \mathrm{kg}^{-1}$. Values are shown for two regions, the polar cap $\left(r<r_{p c}\right)$ and the annulus between the polar cap boundary and zero potential boundary $\left(r_{p c}<r<r_{0}\right)$, two activity levels and three days.

\begin{tabular}{|c|c|c|c|c|c|}
\hline \multicolumn{2}{|c|}{ Day number } & \multicolumn{2}{|l|}{$r<r_{p c}$} & \multicolumn{2}{|c|}{$r_{p c}<r<r_{0}$} \\
\hline & & $\begin{array}{l}\text { Activity } \\
\text { level } 5\end{array}$ & $\begin{array}{l}\text { Activity } \\
\text { level } 9\end{array}$ & \multirow{2}{*}{$\begin{array}{l}\begin{array}{l}\text { Activity } \\
\text { level } 5\end{array} \\
10.1\end{array}$} & \multirow{2}{*}{$\begin{array}{l}\begin{array}{l}\text { Activity } \\
\text { level } 9\end{array} \\
22.5\end{array}$} \\
\hline 355 & $\begin{array}{l}\text { SS } \\
\text { TD }\end{array}$ & $\begin{array}{l}5.12 \\
11.2\end{array}$ & $\begin{array}{l}14.8 \\
29.5\end{array}$ & & \\
\hline 172 & $\begin{array}{l}\text { SS } \\
\text { TD }\end{array}$ & $\begin{array}{l}5.98 \\
9.01\end{array}$ & $\begin{array}{c}7.32 \\
13.1\end{array}$ & $\begin{array}{l}12.7 \\
14.6\end{array}$ & $\begin{array}{l}16.9 \\
22.2\end{array}$ \\
\hline 263 & $\begin{array}{l}\text { SS } \\
\text { TD }\end{array}$ & $\begin{array}{c}7.52 \\
14.7\end{array}$ & $\begin{array}{l}13.9 \\
26.1\end{array}$ & $\begin{array}{l}13.9 \\
17.7\end{array}$ & $\begin{array}{l}21.2 \\
33.0\end{array}$ \\
\hline
\end{tabular}

Fig. 5a-c. Rate of energy input to the F-region (pressure level 12) thermosphere averaged over a 1 -h period measured in $\mathrm{W} \mathrm{kg}^{-1}$. a Steady-state, b time-varying for the same mean conditions as a, c the additional heating due to time-varying field over steady-state. The position of the polar cap boundary (solid and dashed circle) and the continents is shown midway through this period (12.5 UT). Noon local time is at the top of the plots. The colour scale shown by the upper bar is for plots $\mathbf{a}$ and $\mathbf{b}$ and the lower bar is for plot $\mathbf{c}$

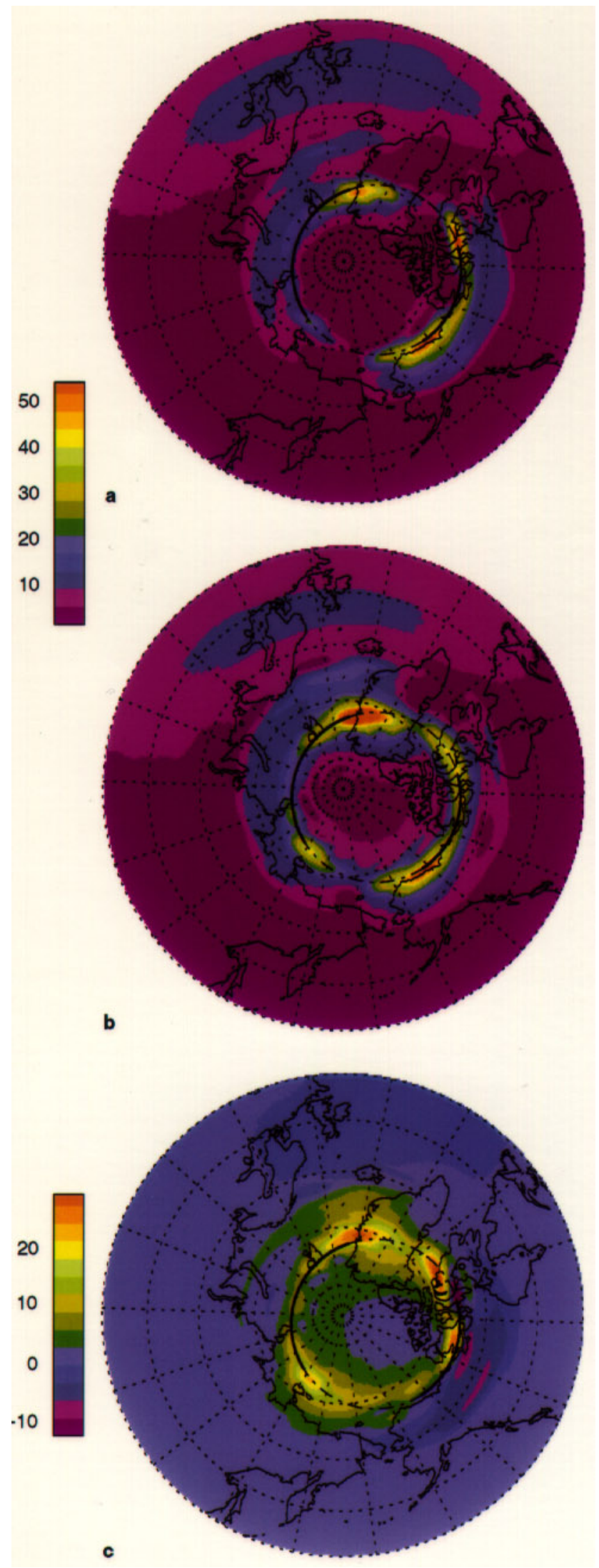


activity level during the northern winter solstice the difference in heating rates is only $30 \%$ in the annulus, while the largest difference $(59 \%)$ in this region is seen at the same time of year but at the higher activity level.

The polar plots in Fig. 5a,b show the mean power dissipated over a 1-h period for the steady-state and time-dependent models respectively, for the lower activity level at winter solstice, while Fig. 5c displays the difference in these two values. Similar plots obtained for the other periods and activity levels show qualitatively the same gross features, indicating that, for both models, most Joule heating occurs near the flux gaps and particularly at the gap edges where ion velocities are very large and the flow characterised by rotational shears. The greatest differences in the two models also occur at the flux gap edges where the time-varying field causes greater heating. Differences are much less in the regions between the polar cap and the zero potential boundary. This appears to be due to there actually being less energy input in the time-dependent model than in the steady-state model in the sectors between the adiaroic boundary segments and the fixed zero potential boundary. This difference may result from the fact that, during time-dependent convection, the polar cap expands and contracts about a mean area equal to that in the steady-state case. This means that the mean radius of the polar cap is actually less in the time-dependent case, the separation of the polar cap boundary and zero potential boundary is greater and thus the mean electric fields in this region are reduced.

Volume integrated Joule heating values have also been obtained for the two models for the region inside the zero potential boundary at altitudes above $120 \mathrm{~km}$. These values are shown in Table 2 and indicate that time-dependent convection would generally be responsible for an additional $25-50 \%$ energy input into this region of the atmosphere.

Comparison of ion density profiles averaged over the polar cap area for the lower activity level (Fig. 6) shows the trend for ion density to increase for the time-dependent model with peak values being some $20 \%$ larger at winter solstice than in the steady-state model. This difference is less at other times, being negligible at summer solstice. This trend is reversed for the higher activity level with a time-varying field reducing ion densities. For both activity levels, however, the effective temperature (which is a function of the ion and nitrogen temperatures and rela-

Table 2. Comparison of steady-state (SS) and time-dependent (TD) total Joule heating rates for the high-latitude Northern Hemisphere region within the zero potential boundary above $120 \mathrm{~km}$ altitude $(\mathrm{GW})$. Values are shown for two activity levels and 3 days

\begin{tabular}{cccc}
\hline Day number & $\begin{array}{c}\text { Activity } \\
\text { level } 5\end{array}$ & $\begin{array}{l}\text { Activity } \\
\text { level } 9\end{array}$ \\
\hline 355 & SS & 5.92 & 53.1 \\
& TD & 7.97 & 71.8 \\
172 & SS & 12.3 & 73.1 \\
& TD & 16.6 & 110.5 \\
263 & SS & 8.25 & 61.0 \\
& TD & 10.2 & 86.2 \\
\hline
\end{tabular}

tive ion-neutral velocity) is increased with a time-varying electric field. Both these results may be explained by considering the rate coefficient, $k_{1}$, for the main plasma loss process at $\mathrm{F}$-region altitudes:

$\mathrm{O}^{+}+\mathrm{N}_{2} \rightarrow \mathrm{N}+\mathrm{NO}^{+}$.

The rate, $k_{1}$, is dependent on the effective temperature and has a minimum value at a temperature of $1050 \mathrm{~K}$. It increases markedly for temperatures greater than this value (St. Maurice and Torr, 1978). In the winter at the lower activity level effective temperatures are generally well below the loss rate minimum. Thus the increase in effective temperature resulting from increased R.M.S. ionneutral relative velocities due to time varying electric fields actually reduces the plasma loss rate. For the lower activity level at summer solstice temperatures are close to, or slightly above, the threshold leaving the ion densities very nearly unchanged in a time-varying field, while at the higher activity level, increased ion temperatures result in increased losses and plasma depletion.

Increased Joule heating observed during time-varying convection results in localised heating of the neutral atmosphere. Transport of heat away from these localised areas means that the mean heights of all pressure levels are increased for the whole of the area defined by the extent of the high-latitude ionospheric convection pattern. It has been found (results not shown) that the mean height increase varies between $1 \mathrm{~km}$ at $120 \mathrm{~km}$ altitude and 20 $\mathrm{km}$ at $400 \mathrm{~km}$ altitude.

Also important in coupling the ionosphere to the neutral atmosphere is momentum transfer whereby plasma drift significantly alters neutral winds. In fact, at F-region altitudes and high latitudes this is the main process by which thermospheric winds are generated. The important mechanism in this respect is that of ion drag (or equivalently electrodynamic forcing). However, pressure differences at fixed heights generated by Joule heating of the atmosphere as described will result in additional horizontal thermospheric winds and increase the momentum transfer of ionospheric convection to the neutral atmosphere.

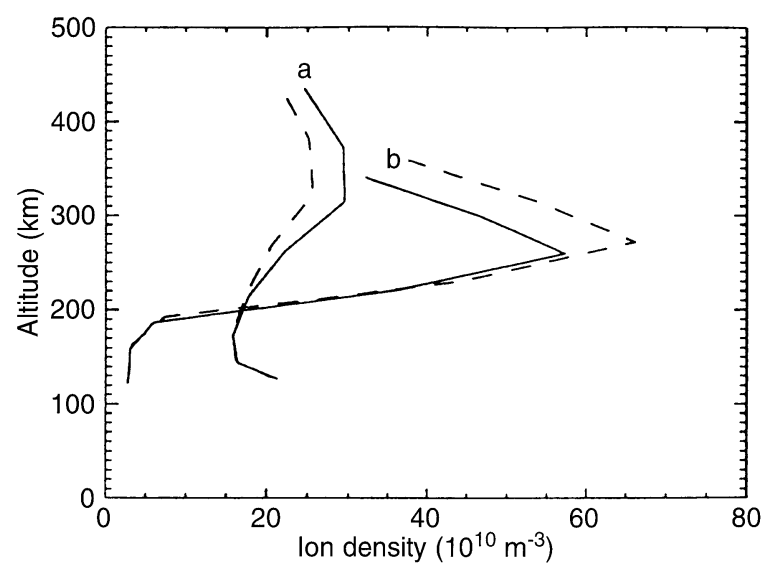

Fig. 6. Mean ion density profiles for the polar cap at summer solstice and high activity level a and winter solstice and low activity level, b. Dashed lines are for time-varying conditions and solid lines for steady-state conditions 
As a measure of the momentum transfer to the thermosphere, the total translational kinetic energy of the neutral winds above a height of $120 \mathrm{~km}$ has been calculated for the two models. These values are shown for comparison in Table 3 for the area inside the polar cap and the annulus between the polar cap boundary and latitude of zero potential. It needs to be emphasised that these winds are not generated by ionospheric drifts alone, and have contributions from thermal winds which would exist in the absence of ionospheric drifts. In places where drift generated winds oppose thermal winds, the former will actually reduce neutral kinetic energy. Thermospheric winds are also generated by the upwards propagation of tidal effects which are imposed at an altitude of approximately $100 \mathrm{~km}$ in the coupled model. These tides are the same in the timedependent and steady-state models. Therefore, although absolute values for the total wind energy will depend on the height above which we integrate, we would nevertheless expect the differences in results for the two models to be qualitatively the same regardless of the exact integration heights. Significant differences are indeed seen between the steady-state and time-dependent model results which must be related to momentum transfer processes. It is seen that the total kinetic energy of the neutral atmosphere is actually reduced in time-varying convection on all 3 days studied and for both activity levels. The difference is greatest for the higher activity level where ion drag forces will dominate neutral atmosphere dynamics. For the higher activity level, the reduction in neutral winds is not unexpected since time-varying fields have been shown to reduce ion concentrations which in turn reduces the ion drag force on the neutrals. Conversely, one might expect the increase in ion concentrations at the lower activity level to actually increase ion drag. However, an explanation can be obtained for the reduction in neutral gas velocities by considering effects close to an adiaroic section of the polar cap boundary during time-dependent convection. A parcel of neutral gas which is stationary at this point will experience the passage of plasma which becomes anti-sunward as the polar cap boundary expands over its location. Some time later, the boundary will contract, so that the parcel lies outside the boundary and

Table 3. Comparison of steady state (SS) and time-dependent (TD) values of total translational kinetic energy of the neutral air wind $\left(10^{12} \mathrm{~J}\right)$. Values are shown for two regions in the Northern Hemisphere, the polar cap $\left(r<r_{p c}\right)$ and the annulus between the polar cap boundary and zero potential boundary $\left(r_{p c}<r<r_{0}\right)$, two activity levels and 3 days.

\begin{tabular}{|c|c|c|c|c|c|}
\hline \multicolumn{2}{|c|}{ Day number } & \multicolumn{2}{|l|}{$r<r_{p c}$} & \multicolumn{2}{|c|}{$r_{p c}<r<r_{0}$} \\
\hline & & $\begin{array}{l}\text { Activity } \\
\text { level } 5\end{array}$ & $\begin{array}{l}\text { Activity } \\
\text { level } 9\end{array}$ & $\begin{array}{l}\text { Activity } \\
\text { level } 5\end{array}$ & $\begin{array}{l}\text { Activity } \\
\text { level } 9\end{array}$ \\
\hline 355 & $\begin{array}{l}\text { SS } \\
\text { TD }\end{array}$ & $\begin{array}{l}4.22 \\
3.96\end{array}$ & $\begin{array}{l}30.8 \\
26.6\end{array}$ & $\begin{array}{l}4.14 \\
3.82\end{array}$ & $\begin{array}{l}36.0 \\
31.3\end{array}$ \\
\hline 172 & $\begin{array}{l}\text { SS } \\
\text { TD }\end{array}$ & $\begin{array}{l}19.1 \\
16.9\end{array}$ & $\begin{array}{l}77.5 \\
67.0\end{array}$ & $\begin{array}{l}17.5 \\
16.6\end{array}$ & $\begin{array}{l}68.5 \\
62.5\end{array}$ \\
\hline 263 & $\begin{array}{l}\text { SS } \\
\text { TD }\end{array}$ & $\begin{array}{l}9.70 \\
8.80\end{array}$ & $\begin{array}{l}53.5 \\
45.9\end{array}$ & $\begin{array}{l}8.35 \\
8.10\end{array}$ & $\begin{array}{l}43.8 \\
39.0\end{array}$ \\
\hline
\end{tabular}

experiences plasma flow which is sunward. The neutral gas has a response time to the ion drag force which is longer than the polar cap cycle period, and thus will respond roughly to the time-averaged ion velocity in this case. It can be seen from this argument that the time averaged ion velocities, and thus the neutral velocities, in the region bounded by the extrema in boundary locations during the time-dependent case will be reduced compared to the steady-state case, this effect being greatest close to the mean location of the polar cap boundary.

\section{Summary}

We have described a fully time-dependent high-latitude ionospheric convection model based on a solution of Laplace's equation. The model allows for an expanding and contracting polar cap boundary which encloses open anti-sunward convecting field lines. The model described has been successfully incorporated into the Sheffield/ UCL/SEL coupled thermosphere/ionosphere/ plasmasphere model and results compared with those obtained with average steady-state conditions. Future improvements to this model will be the inclusion of non-zero $B_{y}$-type flows and flows generated by viscous interaction of the solar wind with plasma on closed field lines. In the simplified treatment described here, precipitation patterns used in the coupled model have simply been scaled in spatial extent during expansion and contraction of the polar cap. More sophisticated time-dependent precipitation models will obviously be required for more detailed quantitative studies of disturbed periods.

The comparisons of steady-state and 1-h time-varying field results for the northern polar cap region may be summarised as follows:

1. At F-region altitudes, the time-dependent model produces enhanced Joule heating compared to the steadystate model inside the polar cap. These enhancements are largest $(120 \%)$ for quiet conditions at winter solstice. In regions close to flux gap edges, this value rises to nearly $150 \%$. The total (volume integrated) increase in energy input to the region of the atmosphere above $120 \mathrm{~km}$ inside the zero potential boundary is generally about $35 \%$. During summer solstice at activity level $9\left(K_{p}=5-\right)$ this value rises to $50 \%$. Thus, steady-state models may significantly underestimate an important energy source in the high-latitude ionosphere.

2. Momentum transfer to the thermosphere is slightly reduced when time variability is taken into account, with total translational kinetic energy of the neutral winds decreasing by about $10 \%$ at altitudes above $120 \mathrm{~km}$. This decrease is due in part to the reduction in the mean ion velocities close to the mean location of the adiaroic boundary sections of the polar cap. This results from the alternating passage of anti-sunward- and sunward-convecting plasma as the polar cap expands and contracts about its mean location.

3. Of all the parameters studied, changes in ion densities are the most dependent on the chosen conditions. During quiet winter time conditions the average polar cap density at the F2 peak actually increases by some 
$20 \%$ during time-varying conditions due to a decrease in plasma loss rates. Conversely when effective ion temperatures are generally higher, for example during summer and in more disturbed periods, time-varying convection reduces average ion density by about $15 \%$.

Acknowledgements. This work has been supported by PPARC under grant GR/K 06112. We thank R. A. Heelis for helpful discussions.

Topical Editor D. Alcaydé thanks A. D. Richmond and G. Rostoker for their help in evaluating this paper.

\section{References}

Crooker, N. U., Dayside merging and cusp geometry, J. Geophys. Res., 84, 951-959, 1979.

Dungey, J. W., Interplanetary magnetic field and the auroral zones, Phys. Rev. Lett., 6, 47-48, 1961.

Foster, J. C., J. M. Holt, R. G, Musgrove, and D. S. Evans, Ionospheric convection associated with discrete levels of particle precipitation, Geophys. Res. Lett., 13, 656-659, 1986.

Friis Christensen, E., Y. Kamide, A. D. Richmond, and S. Matsushita, Interplanetary magnetic field control of high-latitude electric fields and currents determined from Greenland magnetometer data, J. Geophys. Res., 90, 1325-1338, 1985.

Fuller-Rowell, T. J., and D. S. Evans, Height-integrated Pedersen and Hall conductivity patterns inferred from the TIROS-NOAA satellite data, J. Geophys. Res., 92, 7606-7618, 1987.

Hairston, M. R., and R. A. Heelis, Model of the high-latitude ionospheric convection pattern during southward interplanetary magnetic field using DE 2 data, J. Geophys. Res., 95, 2333-2343, 1990

Heelis, R. A., and W. B. Hanson, High-latitude ion convection in the night-time F region, J. Geophys. Res., 85, 1995-2002, 1980.

Heelis, R. A., W. B. Hanson, and J. L. Burch, Ion convection reversals in the dayside cleft, J. Geophys. Res., 81, 3803-3809, 1976.

Heelis, R. A., J. K. Lowell, and R. W. Spiro, A model of the high-latitude ionospheric convection pattern, J. Geophys. Res., 87, 6339-6345, 1982.

Heelis, R. A., J. C. Foster, O. de la Beaujardiere, and J. Holt, Multistation measurements of high-latitude ionospheric convection, J. Geophys. Res., 88, 10111-10121, 1983.

Heppner, J. P., Empirical models of high-latitude electric fields, $J$. Geophys. Res., 82, 115-1125, 1977.

Heppner, J. P., and N. C. Maynard, Empirical high-latitude electric field models, J. Geophys. Res., 92, 4467-4489, 1987.
Holt, J. M., R. H. Wand, J. V. Evans, and W. L. Oliver, Empirical models for the plasma convection at high latitudes from Millstone Hill observations, J. Geophys. Res., 92, 203-212, 1987.

Iijima, T., and T. A. Potemra, Large-scale characteristics of fieldaligned currents associated with substorms, J. Geophys. Res., 83, 599-615, 1978.

Lockwood, M., The excitation of ionospheric convection, J. Atmos. Terr. Phys., 53, 177-199, 1991.

Lockwood, M., S. W. H. Cowley, H. Todd, D. M. Willis, and C. R. Clauer, Ion flows and heating at a contracting polar cap boundary, Planet. Space Sci., 36, 1229-1253, 1988.

Lockwood, M., S. W. H. Cowley, and M. P. Freeman, The excitation of plasma convection in the high-latitude ionosphere, J. Geophys. Res., 95, 7961-7972, 1990.

Meng, C. I., R. H. Holzworth, and S. I. Akasofu, Auroral circle delineating the poleward boundary of the quiet auroral belt J. Geophys. Res., 82, 164-172, 1977.

Millward, G. H., R. J. Moffett, S. Quegan, and T. J. Fuller-Rowell, A coupled thermosphere-ionosphere-plasmasphere model, CTIP, STEP handbook (in press), 1995.

Moses, J. J., G. L. Siscoe, N. U. Crooker, and D. J. Gorney, IMF $B_{y}$ and day-night conductivity effects in the expanding polar cap convection model, J. Geophys. Res., 92, 1193-1198, 1987.

Moses, J. J., G. L. Siscoe, R. A. Heelis, and J. D. Winningham, Polar cap deflation during magnetospheric substorms, J. Geophys Res., 94, 3785-3789, 1989

Moses, J. J., J. A. Slavin, T. L. Aggson, R. A. Heelis, and J. D. Winningham, Modelling ionospheric convection during a major geomagnetic storm on Oct. 22-23, 1981, J. Geophys. Res., 99, 11017-11025, 1994.

Quegan, S., G. J. Bailey, R. J. Moffett and L. C. Wilkinson, Universal time effects on plasma convection in the geomagnetic frame, $J$. Atmos. Terr. Phys., 48, 25-40, 1986.

Richmond, A. D., Assimilative mapping of ionospheric electrodynamics, Adv. Space Res., 12, 59-68, 1992.

Reiff, P. H., and J. L Burch, IMF $B_{y}$-dependent plasma flow and Birkeland currents in the dayside magnetosphere 2. A global model for northward and southward IMF, J. Geophys. Res., 90, 1592-1609, 1985.

Rostoker, G., D. Savoie, and T. D. Phan, Response of magnetosphere-ionosphere current systems to changes in the interplanetary magnetic field, J. Geophys. Res., 93, 8633-8641, 1988.

Siscoe, G. L., and T. S. Huang, Polar cap inflation and deflation, $J$. Geophys. Res., 90, 543-547, 1985.

St. Maurice, J. P., and D. G. Torr, Non-thermal rate coefficients in the ionosphere: The reactions of $\mathrm{O}^{+}$with $\mathrm{N}_{2}, \mathrm{O}_{2}$ and $\mathrm{NO}, J$ Geophys. Res., 83, 969-977, 1978.

Wolf, R. A., Ionosphere-magnetosphere coupling, Space Sci. Rev., 17, 537-562, 1975. 